\title{
Optimal Trade Execution under Jump Diffusion Process: A Mean-VaR Approach
}

\author{
Tianmin Zhou $\mathbb{D}$, Can Jia, and Handong $\mathrm{Li} \mathbb{D}$ \\ School of Systems Science Beijing Normal University, Beijing 100875, China \\ Correspondence should be addressed to Tianmin Zhou; lsdzdxt_28@hotmail.com and Handong Li; lhd@bnu.edu.cn
}

Received 2 February 2018; Revised 3 May 2018; Accepted 8 May 2018; Published 1 October 2018

Academic Editor: Xiaohua Ding

Copyright (c) 2018 Tianmin Zhou et al. This is an open access article distributed under the Creative Commons Attribution License, which permits unrestricted use, distribution, and reproduction in any medium, provided the original work is properly cited.

\begin{abstract}
In the classical optimal execution problem, the basic assumption of underlying asset price is Arithmetic Brownian Motion (ABM) or Geometric Brownian Motion (GBM). However, many empirical researches show that the return distribution of assets may have heavy tails than those of normal distribution. The uncertain information impact on financial market may be considered as one of the main reasons for heavy tails of return distribution. To introduce this information impact, our paper proposes a Jump Diffusion model for optimal execution problem. The jumps in our model are described by the compound Poisson process where random jump amplitude depicts the information impact on price process. In particular, the model is simple enough to derive closed-form strategies under risk neutral and Mean-VaR criterion. Simulation analysis of the model is also presented.
\end{abstract}

\section{Introduction}

The algorithmic trading in equities and other asset classes has been greatly developed over the past decade. The key problem of algorithmic trading is to decrease execution cost, which is the difference in the value between the ideal trade and what was actually done [1]. The execution cost can be decomposed into explicit costs and implicit costs [2]. Explicit costs are the direct costs of trading, such as commissions, fees, and taxes. Implicit costs are indirect costs, which are mainly due to price impact of trading and quite significant in large trade [3]. To decrease price impact, traders can break up a large trade into a number of smaller blocks. However, a longer execution horizon may increase the exposure to the timing risk.

In pair of seminal works, Bertsimas and Lo [4] first obtained a closed-form solution of optimal execution problem in discrete time. They defined the information price model under the assumption that the unaffected price process follows ABM and added price impact on the execution price of the trade. Almgren and Chriss $[5,6]$ extended the work by taking a tradeoff between expect execution cost and risk. In their trading model, a closed-form solution to the optimal execution problem is given in continuous time, and the price impact is divided into temporary impact and permanent impact. Temporary impact mainly comes from supply-demand imbalances at the moment of trading. In contrast, permanent impact refers to a price change persisting for the whole period. Both temporary and permanent impact function are considered as linear in trade size.

Based on the pioneering works, there is a large number of literatures on the optimal execution problem. Schied and Schöneborn [7] obtained the optimal execution strategy through utility maximization. Gatheral and Schied [8] derived the closed-form solution under the assumption of using GBM as unaffected price process. In their corresponding model, the time-averaged VaR is chosen to quantify the risk associated with trade. Related work along this line with robust solution can be found in Schied [9]. In contrast to the traditional price impact mode, Forsyth [10] considered that the price impact function depended on both trade size and current price, and the numerical method was used in solving mean-variance problem. Forsyth et al. [11] suggested that using quadratic variation as risk measure was time-consistent in the dynamic programming. Cheng et al. [12] summarized the risk criterions and obtained closed-form strategies with uncertain order fills in Almgren-Chriss framework.

A large number of literatures about optimal execution are mainly based on the assumption of ABM or GBM. The 
assumption fails to capture the information impact on the asset price. The information impact includes changing market condition and sudden availability of some information about the asset. There are relatively few studies on the optimal execution problem under the information impact. Bertsimas and Lo [3] first used first-order autoregressive behavior to describe the information impact on unaffected price process. Huberman and Stanzl [13] extended the work by incorporating uncertainty liquidity information. Apart from these studies, few literatures focus on the jump phenomenon in optimal execution problem. The phenomenon describes that the asset price process is often interrupted by some sudden information. These interruptions cause significant discontinuity of asset prices process, the so-called jump phenomenon [14-16], which explains that the asset return probability distribution has heavy tails [17-19]. Moazeni et al. [3] first studied the optimal execution problem under Jump Diffusion process and used Monte Carlo simulation to obtain the minimum CVaR (conditional value at risk) execution strategy. In their model, the compound Poisson process is used to explain the price impact of other large trades. In contrast, we use the compound Poisson process to depict the uncertain information impact and derive the analytic expression for the $\mathrm{VaR}$ (value at risk) evaluation. Then we use discrete stochastic dynamic programming method introduced by Bellman [20] and Bertsimas and Lo [4] to obtain a closed-form solution to optimal execution problem under risk neutral and Mean-VaR criterion.

The remainder of this paper is organized as follows. In Section 2, we introduce the basic definitions of optimal execution problems. Section 3 derive closed-form solutions for the optimal execution strategies under risk neutral criterion and Mean-VaR criterion. We provide the influence of different parameters on the optimal strategies and carry out the Monte Carlo simulation to compare different execution cost in Section 4. The last section concludes some remarks of our model, and the proofs are given in the appendix.

\section{Problem Description and Notations}

This paper assumes that we hold large shares $X$ of an asset which we plan to completely sell over a fixed time interval $[0, T]$. We divide $[0, T]$ into $N$ intervals of length $\Delta t=T / N$ and let $t_{0}=0<t_{1}<\cdots<t_{N}=T$, where $t_{k}-t_{k-1}=\Delta t$ for $k=1,2, \ldots, N$. During the transaction, the deterministic initial asset price is denoted by $S_{0}$, and the dynamics of $S_{k}$ include two distinct components: the Brownian motion and the compound Poisson process. Then the law of motion for $S_{k}$ is expressed as

$$
S_{k}=S_{k-1}+\sigma \sqrt{\Delta t} \varepsilon_{k}+\Delta J_{k}, \quad k=1,2, \ldots, N,
$$

where $\sigma$ represents the volatility of the asset, $\varepsilon_{k}$ is independent random variables with zero mean and unit variance, and $\sigma \sqrt{\Delta t} \varepsilon_{k}=B_{k}-B_{k-1}=\Delta B_{k}$ is the increment of Brownian motion. The uncertain information impact $\triangle J_{k}=\sum_{i=1}^{\triangle P_{k}} Y_{i}$ is the compound Poisson process with the arrival rate $\lambda \triangle t \geq 0$ and the jump amplitude $Y_{i} \sim N\left(\mu_{j}, \sigma_{j}^{2}\right)$, where $\Delta P_{k}$ is the jump number count on $\left(t_{k-1}, t_{k}\right]$.
We specify the trade list $x_{1}, \ldots, x_{N}$ which represent the number of the shares that we seek to sell between times $t_{k-1}$ and $t_{k}$. Let $W_{k}$ denote the holding shares at time $t_{k}$ and satisfy the following expression: $W_{k}=W_{k-1}-x_{k-1}$. We assume that our initial holding shares are $W_{1}=X$ and final holding shares are $W_{N+1}=0$. Similar to Bertsimas and Lo [4], the discrete price process with permanent impact can be expressed as

$$
S_{k}=S_{k-1}-\gamma x_{k}+\sigma \sqrt{\Delta t} \varepsilon_{k}+\Delta J_{k}
$$

where $\varepsilon_{k}$ satisfies $\mathbb{E}_{k}\left[\varepsilon_{k}\right]=\mathbb{E}\left[\varepsilon_{k} \mid S_{k-1}, x_{k}\right]=0, \mathbb{E}_{k}\left[\Delta J_{k}\right]=$ $\mathbb{E}\left[\Delta J_{k} \mid S_{k-1}, x_{k}\right]=\lambda \Delta t \mu_{j}$ and $\gamma$ is the linear permanent impact coefficient which measures the permanent impact of trade size. In addition to permanent impact, the execution price may decrease between $t_{k-1}$ and $t_{k}$ due to temporary imbalances in supply and demand, and the effect does not appear in the next price $S_{k+1}$. According to the Linearpercentage temporary price impact model [4] and temporary market impact model [6], we assume that the temporary impact is the function of trading rate and only affects the execution price during the interval $t_{k-1}$ to $t_{k}$. The execution price $\widetilde{S}_{k}$ is given by

$$
\widetilde{S}_{k}=S_{k}-\eta \frac{x_{k}}{\Delta t}=S_{k}-\tilde{\eta} x_{k}
$$

where $\eta$ represents the linear temporary impact and $\tilde{\eta}=\eta / \Delta t$.

Under the assumption above, we define that the total amount at the end of the time horizon $T$ is $\sum_{k=1}^{N} \widetilde{S}_{k} x_{k}$. The main objective function is to maximize the expected total amount concerned with the given risk measure. Hence the objective function in general form can be described as follows:

$$
\begin{array}{ll}
\max _{x_{k}} & \mathbb{E}_{1}\left[\sum_{k=1}^{N} \widetilde{S}_{k} x_{k}-\rho_{\alpha}\left(\triangle S_{k} W_{k}\right)\right] \\
\text { s.t. } & \sum_{k=1}^{N} x_{k}=X
\end{array}
$$

where $\rho_{\alpha}(\bullet)$ is the risk measure of execution under the risk aversion parameter $\alpha \in[0,1]$ and $\Delta S_{k}=S_{k}-S_{k-1}$ is the unaffected price increment in (1). We compute the risk measure associated with $W_{k}$ at each time point. In next section, we first consider the optimization problem under risk neutral and then take the execution risk into the optimization.

\section{Optimal Solution of Problems}

In this section, the optimizations under two conditions will be described in detail.

3.1. Optimal Execution Strategy under Risk Neutral. We first maximize the expected total amount at the end of the time 
horizon $T$ and our optimization problem can be described as follows:

$$
\begin{array}{ll}
\max _{x_{k}} & \mathbb{E}_{1}\left[\sum_{k=1}^{N} \widetilde{S}_{k} x_{k}\right] \\
\text { s.t. } & \sum_{k=1}^{N} x_{k}=X,
\end{array}
$$

which aims to find an execution strategy $\left\{x_{k}, k=1, \ldots, N\right\}$ to maximize the expected total amount. The trading strategy needs to completely sell total shares at time $T$. Then we get a typical optimal control problem, and we can solve (5) by the discrete stochastic dynamic programming method and the closed-form solution can be expressed as follows:

$$
\begin{aligned}
x_{N-k}^{*}= & \frac{W_{N-k}}{k+1}-\frac{k \lambda \Delta t \mu_{j}}{2(\gamma+2 \widetilde{\eta})}, \quad k=0,1, \ldots, N-1, \\
V_{N-k}= & W_{N-k}\left(S_{N-k-1}-\frac{\gamma(k+2)+2 \widetilde{\eta}}{2(k+1)} W_{N-k}\right. \\
& \left.+\frac{(k+2) \lambda \Delta t \mu_{j}}{2}\right)+\frac{a_{k}\left(\lambda \Delta t \mu_{j}\right)^{2}}{2(2 \gamma+4 \widetilde{\eta})},
\end{aligned}
$$

where $a_{k}=a_{k-1}+(1+k) k / 2, a_{0}=0, x_{N-k}^{*}$ is the optimal execution strategy, $V_{N-k}$ is the optimal-value function and $W_{N-k}$ is the number of holding shares for $k=0,1, \ldots, N-1$. The corresponding proof is provided in Appendix A.

When $\lambda=0$, the optimal execution strategy turns to

$$
x_{N-k}^{*}=\frac{W_{N-k}}{k+1},
$$

and the optimal-value function is

$$
\begin{aligned}
& V_{N-k}\left(S_{N-k-1}, W_{N-k}\right) \\
& \quad=W_{N-k}\left(S_{N-k-1}-\frac{\gamma(k+2)+2 \widetilde{\eta}}{2(k+1)} W_{N-k}\right) .
\end{aligned}
$$

Substituting the initial conditional $W_{1}=X$ into (8) yields optimal execution strategy size $x_{1}^{*}=W_{1} / N$. By the recursive law, we can get

$$
x_{1}^{*}=x_{2}^{*}=\cdots=x_{N}^{*}=\frac{X}{N}
$$

which shows that total shares are sold at a constant rate over the time horizon $T$. The optimal execution strategy is called TWAP (Time Weighted Average Price) or Naïve strategy, and the remainder shares are $W_{N-k}=W_{1}-\sum_{i=1}^{N-k-1} x_{i}^{*}=(k+$ 1) $X / N$. Under the linear impact function, the TWAP strategy has the least price impact during transaction.

When $\lambda \neq 0$, in contrast to the TWAP strategy, the optimal execution strategy (6) depends on the price impact and expected jump size per unit time. While the TWAP strategy never buys for sell execution, the optimal execution strategy may include buying in some periods, and this is also provided in literature [3]. For $\mu_{j}>0$, the decrease speed of the holding shares is smaller than TWAP strategy at the beginning of the trade, and the rate is larger than the TWAP strategy near the expiration. For $\mu_{j}=0$, the optimal execution strategy reduces to the TWAP strategy. For $\mu_{j}<0$, the decrease speed of the holding shares is larger than TWAP strategy at the beginning of the trade and the rate is smaller than the TWAP strategy near the expiration.

3.2. Optimal Execution Strategy under Mean-VaR. Apart from the expected execution amount, a risk-averse investor also needs to consider the execution risk. He will take a tradeoff between price impact and risk. That is to say, investors always want to pursue a strategy that maximizes the expected execution amount under a given risk preference. Therefore, we add a risk measure into the objective function and the optimized objective function becomes

$$
\begin{aligned}
\max _{x_{k}} & \mathbb{E}_{1}\left[\sum_{k=1}^{N} \widetilde{S}_{k} x_{k}-\operatorname{VaR}\left(\Delta S_{k}\right) W_{k}\right] \\
\text { s.t. } & \sum_{k=1}^{N} x_{k}=X
\end{aligned}
$$

where $\operatorname{VaR}_{\alpha}\left(\triangle S_{k}\right)=\inf \left\{l \in \mathbb{R}: P\left(-\triangle S_{k}>l\right) \leq\right.$ $1-\alpha\}$ is the risk exposure of our holding shares $W_{k}$ at confidence level $\alpha \in(0,1)$. Under the assumption of Jump Diffusion process, $\operatorname{VaR}_{\alpha}\left(\triangle S_{k}\right)=-\Phi_{\triangle S_{k}}^{-1}(\alpha)$, and $\Phi_{\triangle S_{k}}(\bullet)$ is the cumulative distribution function of unaffected price increment in (1), which can be expressed as

$$
\Phi_{\triangle S_{k}}(x)=\int_{-\infty}^{x} f_{\triangle S_{k}}(y) d y
$$

Here $f_{\triangle S_{k}}(\bullet)$ is a Gaussian Mixture Model (GMM). A proof for (12) is given in Appendix B. Next, the closed-form solution of problem (11) is

$$
\begin{aligned}
x_{N-k}^{*} & =\frac{W_{N-k}}{k+1}-\frac{k\left(\lambda \Delta t \mu_{j}-V a R_{\alpha}\left(\triangle S_{1}\right)\right)}{2(\gamma+2 \widetilde{\eta})}, \\
\quad k=0,1, \ldots, N-1, & \\
V_{N-k} & =W_{N-k}\left(S_{N-k-1}-\frac{\gamma(k+2)+2 \widetilde{\eta}}{2(k+1)} W_{N-k}\right. \\
+ & \left.\frac{(k+2)\left(\lambda \triangle \mu_{j}-V a R_{\alpha}\left(\triangle S_{1}\right)\right)}{2}\right) \\
+ & \frac{a_{k}\left(\lambda \triangle \mu_{j}-V a R_{\alpha}\left(\triangle S_{1}\right)\right)^{2}}{2(2 \gamma+4 \widetilde{\eta})},
\end{aligned}
$$

where $a_{k}=a_{k-1}+(1+k) k / 2, a_{0}=0, x_{N-k}^{*}$ is the optimal execution strategy, $V_{N-k}$ is the optimal-value function, and $W_{N-k}$ is the number of holding shares for $k=0,1, \ldots, N-1$. We provide a corresponding proof in Appendix C. 
TABLE 1: Simulation parameters.

\begin{tabular}{ll}
\hline Parameters & Values \\
\hline Initial price $S_{0}$ & $\$ 100 /$ share \\
Total position $X$ & $10^{6}$ shares \\
Trading time $T$ & 1 day \\
The permanent impact coefficient $\gamma$ & $5 \times 10^{-7} \$ / \mathrm{share}^{2}$ \\
The temporary impact coefficient $\eta$ & $5 \times 10^{-6}(\$ \cdot \Delta t) / \mathrm{share}^{2}$ \\
The daily volatility $\sigma$ & $1\left(\$ / \mathrm{share}^{2} / \Delta t^{1 / 2}\right.$ \\
The number of trades $N$ & 240 \\
The interval between trade $\Delta t$ & $1 / 240$ \\
Arrival rate of jump $\lambda$ & {$[0,50]$} \\
Mean of jump amplitude $\mu_{j}$ & {$[-0.5,0.5]$} \\
Variance of jump amplitude $\sigma_{j}$ & {$[0.01,0.5]$} \\
Appetite for risk $\alpha$ & {$[0.01,0.5]$} \\
\hline
\end{tabular}

When $\lambda=0$, the unaffected price process obeys the ABM, and the optimal execution strategy is

$$
x_{N-k}^{*}=\frac{W_{N-k}}{k+1}+\frac{k V a R_{\alpha}\left(\triangle B_{1}\right)}{2(\gamma+2 \widetilde{\eta})},
$$

where $\triangle B_{1}$ is the increment of Brownian motion. The optimal-value function is

$$
\begin{aligned}
V_{N-k}= & W_{N-k}\left(S_{N-k-1}-\frac{\gamma(k+2)+2 \widetilde{\eta}}{2(k+1)} W_{N-k}\right. \\
& \left.-\frac{(k+2) V_{a}\left(\triangle B_{1}\right)}{2}\right) \\
& +\frac{a_{k}\left(V a R_{\alpha}\left(\triangle B_{1}\right)\right)^{2}}{2(2 \gamma+4 \widetilde{\eta})} .
\end{aligned}
$$

The optimal execution strategy depends on the price impact and the risk exposure of our holding shares. The decrease speed of the holding shares is larger than TWAP strategy at the beginning of the trade, and the rate is smaller than the TWAP strategy near the expiration.

When $\lambda \neq 0$, the unaffected price process obeys the Jump Diffusion process, and the optimal execution strategy is (13). Because of adding jump into ABM, the optimal execution strategy depends on $\operatorname{VaR}_{\alpha}\left(\triangle S_{1}\right)$ and $\lambda \triangle t \mu_{j}$. For $\lambda \triangle t \mu_{j}-$ $\operatorname{VaR}_{\alpha}\left(\triangle S_{1}\right)>0$, the expectation of the compound Poisson process covers the risk exposure, and the optimal execution strategy trades slowly except near expiration. The optimal execution strategy reduces to the TWAP strategy, for $\lambda \Delta t \mu_{j}-$ $\operatorname{VaR}_{\alpha}\left(\triangle S_{1}\right)=0$. For $\lambda \triangle t \mu_{j}-\operatorname{VaR}_{\alpha}\left(\triangle S_{1}\right)<0$, the optimal execution strategy trades quickly except near expiration. The comparison among these strategies is illustrated in next section.

\section{Numerical Simulation}

In this section, we first illustrate the influences of parameters variations on optimal execution strategies. Then we show the comparison among transaction costs of different execution strategies. The initial parameters are given as follows.
We have a single asset with current market price $S_{0}=\$ 100$, and the initial holdings are $X=10^{6}$ shares. The whole shares are expected to sell in one day $T=1$ and the interval between trade is $\Delta t=1 / 240$. The daily volatility of stock is $\sigma=1$, the volatility in every time interval is $1 / \sqrt{240}$, the temporary impact coefficient is $\eta=5 \times 10^{-6}$, and the permanent impact coefficient is $\gamma=5 \times 10^{-7}$. The range of arrival rate is $\lambda \epsilon$ $[0,50]$ and the jump amplitude is the normal distribution with $\mu_{j} \in[-0.5,0.5]$ and $\sigma_{j} \in[0.01,0.5]$, and the range of preference for risk is $\alpha \in[0.01,0.5]$ (see Table 1 ).

4.1. Numerical Result under Risk Neutral. The optimal execution strategy varying with the parameter $\mu_{j}$ is shown in Figure 1, the plot is generated by using parameters chosen as in Table 1. Each curve represents a distinct strategy. The blue solid line indicates the optimal solution for parameters $\mu_{j}=0.4$ and $\lambda=50$. The solution postpones selling because of positive expected return $\mu_{j}$. The blue dotted line has $\mu_{j}=0$ and $\lambda=50$ and the optimal execution strategy reduces to the TWAP strategy. The light blue solid has $\mu_{j}=-0.4$ and $\lambda=50$, and the optimal execution strategy sells quickly to reduce the exposure to the negative return $\mu_{j}$. That is to say, when the expected jump amplitude is positive, the strategy intends to hold the stock and waits for an appropriate time to sell. Otherwise, we sell the holdings quickly to lock in prices.

4.2. Numerical Result with Mean-VaR Strategy. The optimal execution strategy under ABM is well-studied. Figure 2(a) illustrates the comparison between ABM and Jump Diffusion process with parameters $\lambda=25, \mu_{j}=-0.05$ and $\sigma_{j}=$ 0.05. Figure 2(b) shows the comparison of holding shares during execution between different price processes. The blue solid line is optimized under ABM and the light blue line is optimized under Jump Diffusion process. From the figure, we can see that the execution strategy under Jump Diffusion process sells more quickly than that under ABM.

Next, we analyze the influence of parameters variations on optimal strategy. For $\sigma_{j} \ll \sigma$, the variance of $\triangle S_{k}$ is mainly affected by parameters $\lambda, \mu_{j}$, and $\alpha$. So we fix $\sigma_{j}$ in simulation analysis. In Figure 3 , the surfaces are generated by using 


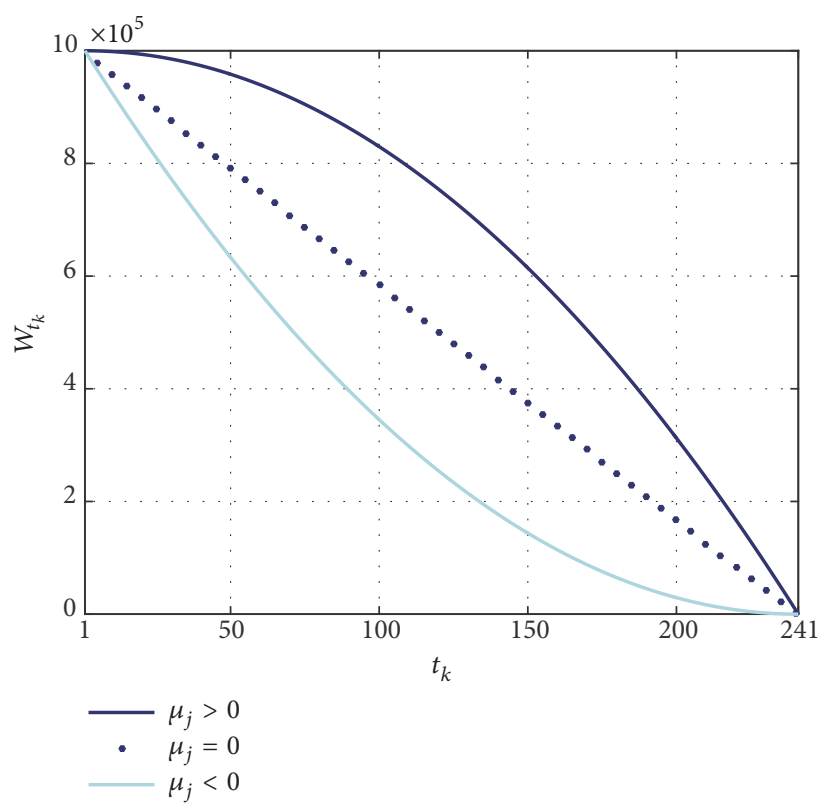

FIGURE 1: The execution trajectories under different $\mu_{j}$.

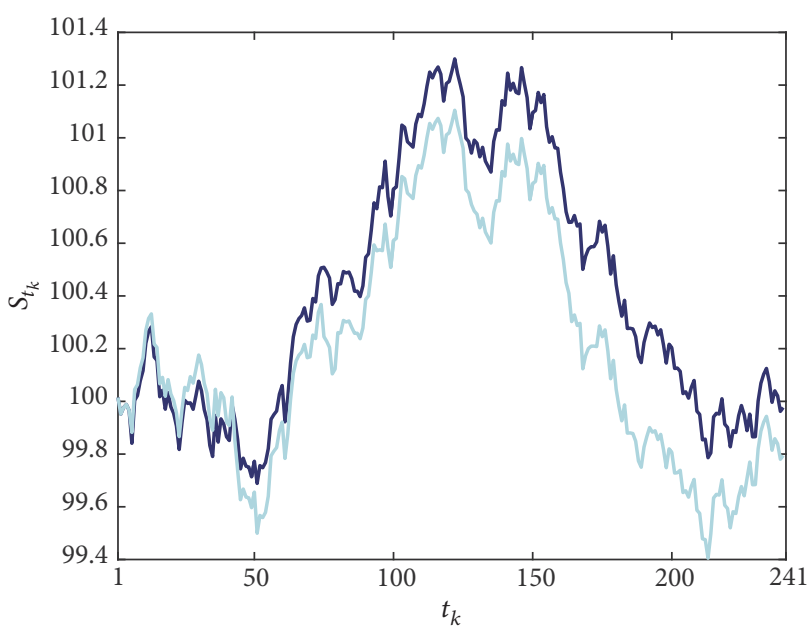

ABM

Jump Diffusion

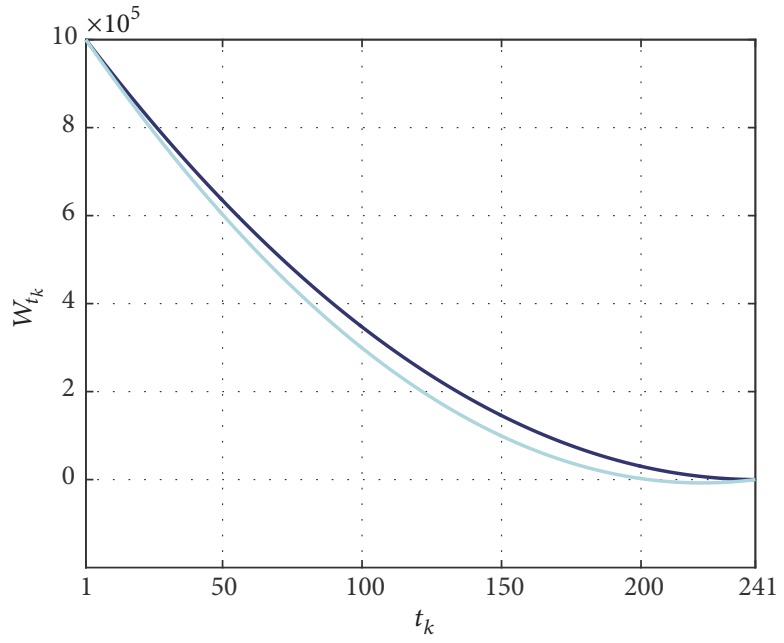

$\lambda=0, \alpha=0.1$ $\lambda>0, \alpha=0.1$

(a)

(b)

FIgURE 2: The execution trajectories under different price processes.

parameters as $\lambda \in\{5,50\}, \sigma_{j}=0.05, \mu_{j} \in[-0.5,0.5]$. When $\lambda=5$, the execution trajectory is sensitive to the change of $\alpha$. When $\alpha$ decreases, we sell quickly at the beginning of the trade to reduce exposure to the volatility risk. When $\lambda=50$, the execution trajectory is sensitive to the change of $\alpha$ and $\mu_{j}$, and there are obvious differences among the optimal strategies. The trading style changes from risk-averse to risk preference with $\mu_{j}$ increasing. The changes mentioned above are caused by $\lambda \triangle t \mu_{j}-\operatorname{VaR}_{\alpha}\left(\triangle S_{1}\right)$ in (13). If $\lambda \Delta t \mu_{j}-$ $\operatorname{VaR}_{\alpha}\left(\triangle S_{1}\right)<0$, the optimal execution strategy sells quickly.
If $\triangle t \mu_{j}-\operatorname{VaR} R_{\alpha}\left(\triangle S_{1}\right)=0$, the strategy reduces to the TWAP strategy. If $\lambda \Delta t \mu_{j}-\operatorname{VaR}_{\alpha}\left(\triangle S_{1}\right)>0$, the strategy postpones selling.

4.3. Numerical Result of Simulated Trading. Assume that the stock price process follows the Jump Diffusion process, the temporary impact and permanent impact are linear function of trading size for each time interval. Let the price processes $S_{k}=S_{k-1}+\sigma \sqrt{\Delta t} \varepsilon_{k}+\Delta J_{k}$ be unaffected asset price process. 

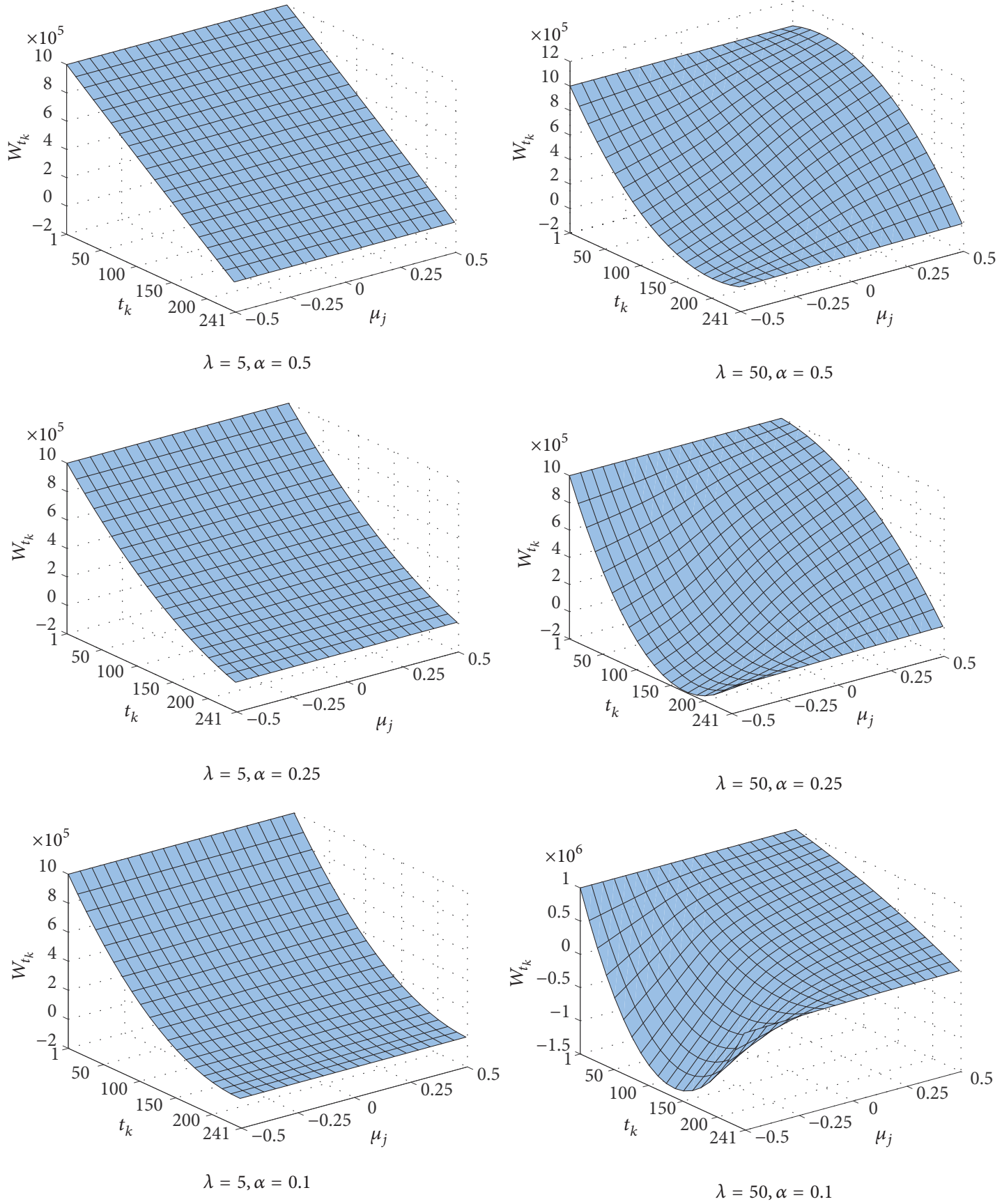

FIGURE 3: The influence of parameters $\mu_{j}$ and $\alpha$ on execution trajectories.

We specify that the execution strategy is the simple sell behavior, the number of the shares is one million $\left(X=10^{6}\right)$, and the execution time is one day. Under the simulation mechanism above, we compare the 5 strategies: Random strategy (random shares-split), TWAP strategy, Risk neutral strategy, Mean-VaR strategy, and Mean-VaR ${ }_{\mathrm{jd}}$ (price with jumps) strategy. We reset $\lambda, \mu_{j}, \sigma_{j}$, and $\alpha$ to $25,-0.5,0.5$, and 0.1 which describe the volatile market. The total cost of trading is the difference between the ideal trade and what was actually done [1], and we use $C_{0}=S_{0} X-\sum_{k=1}^{N} \widetilde{S}_{k} x_{k}$ to measure the transaction cost for Random strategy, TWAP strategy, and Risk neutral strategy. Since risk consideration is given in deriving optimal execution strategies, we use $C_{1}=S_{0} X-\sum_{k=1}^{N} \widetilde{S}_{k} x_{k}+\sum_{k=1}^{N} W_{k} \operatorname{VaR}_{90 \%}\left(\triangle S_{k}\right)$ to measure the transaction cost for Mean-Risk strategies. Then 5000 samples for 5 strategies are drawn by Monte Carlo simulation and the results are as shown in Table 2.

From the results, we find that the random strategy has the largest cost. Because of taking jump process into consideration, the Risk neutral strategy and the Mean- $\mathrm{VaR}_{\mathrm{jd}}$ strategy 
TABLE 2: Optimal execution results.

\begin{tabular}{lr}
\hline Strategy & Average of cost \\
\hline Random & $\$ 13,199,249.89$ \\
TWAP & $\$ 11,569,787.27$ \\
Risk neutral & $\$ 10,871,389.31$ \\
Mean-VaR & $\$ 21,321,881.81$ \\
Mean-VaR ${ }_{\text {dd }}$ & $\$ 19,111,757.60$ \\
\hline
\end{tabular}

TABLE 3: Optimal trading results.

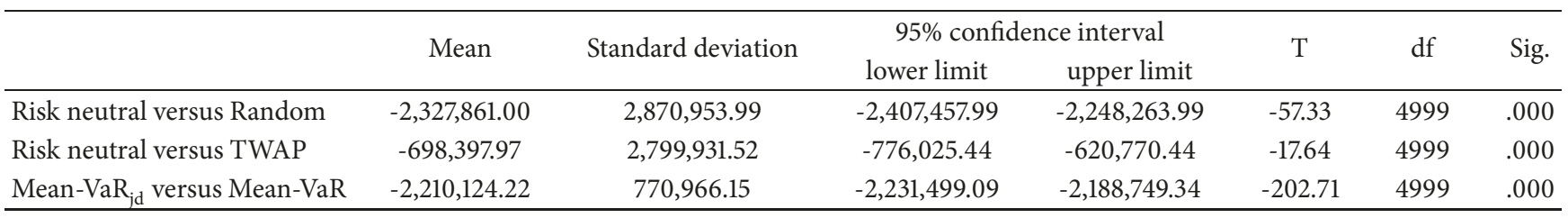

have smaller transaction cost than the TWAP strategy and Mean-VaR strategy, respectively. That is to say, if there is the jump in the price process, the optimal execution strategy is different from that under ABM.

To compare the differences among these costs in statistics, we carry out a paired-samples t-test to analyze the distinction of the transaction costs. The paired-samples t-test is widely used for determining whether there is a systematic deviation between paired test data; that is, if the difference between paired test data is significant, the difference can be always observed under different conditions. Since the observation data are from different objects, they are not sample data, and the test method is as follows.

Considering $n$ pairs of independent observation data $\left(X_{1}, Y_{1}\right),\left(X_{2}, Y_{2}\right), \ldots,\left(X_{n}, Y_{n}\right)$, their differences are described as $D_{1}=X_{1}-Y_{1}, D_{2}=X_{2}-Y_{2}, \ldots, D_{n}=X_{n}-Y_{n}$, respectively; thus, $D_{i} \sim N\left(\mu_{D}, \sigma_{D}^{2}\right)$ for $i=1,2, \ldots, n$. If $\mu_{D}$ and $\sigma_{D}^{2}$ are unknown, then the null hypothesis is tested as follows: $H_{0}: \mu_{D}=0 ; H_{1}: \mu_{D} \neq 0$. The t-test statistics and rejection regions used in this study are obtained by $|t|=\left|\bar{D} /\left(S_{D} / \sqrt{n}\right)\right|$ and $\bar{D}, S_{D}$, and $\alpha$ are the sample mean, the sample standard deviation, and the significance level, respectively.

Then we use $5 \times 5000$ transaction costs by Monte Carlo simulation to construct the paired data, and to identify that if there exists an obvious differences in them, and the results are as shown in Table 3.

From Table 3, we can see the results of paired t-test for transaction costs, which show that there are significant differences between paired transaction costs. The Risk neutral strategy has a smaller transaction cost than the TWAP strategy and Random strategy in our simulations. The Mean-VaR $\mathrm{R}_{\mathrm{jd}}$ also has a smaller transaction cost than Mean-VaR. To sum up, execution of large shares by our strategy can effectively reduce the transaction costs in our simulations.

\section{Conclusion}

Most published literatures on the optimal execution problem are typically solved in Arithmetic Brownian Motion or Geometric Brown motion. In this paper, we use Jump Diffusion process to capture the uncertain information impact, which reflects more realistic phenomenon of modern financial market. The proposed model includes a compound Poisson process and a closed-form solution to the optimal execution strategy is derived by stochastic dynamic programming method. The optimal execution strategy does not depend on the asset price volatility under risk neutral. In contrast, under Mean-VaR, the optimal execution strategy depends on price distribution. In addition, we illustrate the influence of parameters changes on the optimal execution strategy. The paired $t-$ test is applied to perform statistical analysis and shows that the paired execution costs are obviously different. Traders can determine an optimal execution strategy according to an appropriate optimization model. Although we provide a closed-form solution to the optimal execution strategy, there are many issues that need to be modified. For example, our optimization does not incorporate a nonnegative constrain, Jump Diffusion process in our model may give negative value, etc. Therefore, various further works need to be extended.

\section{Appendix}

\section{A. Proof of (6) and (7)}

According to the iterative form of the value function of dynamic programming, we can get

$$
V_{k}\left(S_{k-1}, W_{k}\right)=\max _{x_{k}} \mathbb{E}_{k}\left[\widetilde{S}_{k} x_{k}+V_{k+1}\left(S_{k}, W_{k+1}\right)\right]
$$

and, by staring at the period $N$, we obtain

$$
\begin{aligned}
V_{N} & \left(S_{N-1}, W_{N}\right)=\max _{x_{N}} \mathbb{E}_{N}\left[\widetilde{S}_{N} W_{N}\right] \\
= & \left(S_{N-1}-\gamma W_{N}+\lambda \Delta t \mu_{j}-\tilde{\eta} W_{N}\right) W_{N} .
\end{aligned}
$$

Since this is the last period and $W_{N+1}$ must to be set to 0 , there is no choice but to execute the entire remaining order $W_{N}$. 
In the next-to-last period $N-1$, the Bellman iteration is as follows:

$$
\begin{aligned}
& V_{N-1}\left(S_{N-2}, W_{N-1}\right)=\max _{x_{N-1}} \mathbb{E}_{N-1}\left[\widetilde{S}_{N-1} x_{N-1}\right. \\
& \left.+V_{N}\left(S_{N-1}, W_{N}\right)\right]=\max _{x_{N-1}} \mathbb{E}_{N-1}\left[\left(S_{N-2}-\gamma x_{N-1}\right.\right. \\
& \left.+\sigma \sqrt{\triangle t} \varepsilon_{N-1}+\Delta J_{N-1}-\tilde{\eta} x_{N-1}\right) x_{N-1}+V_{N}\left(S_{N-2}\right. \\
& \left.\left.-\gamma x_{N-1}+\sigma \sqrt{\Delta t} \varepsilon_{N-1}+\Delta J_{N-1}, W_{N-1}-x_{N-1}\right)\right] .
\end{aligned}
$$

The conditional expectation of the above equation at period $N-1$ is

$$
\begin{aligned}
& V_{N-1}\left(S_{N-2}, W_{N-1}\right)=\max _{x_{N-1}}\left[\left(S_{N-2}-\gamma x_{N-1}+\lambda \Delta t \mu_{j}\right.\right. \\
& \left.-\tilde{\eta} x_{N-1}\right) x_{N-1}+\left(S_{N-2}-\gamma x_{N-1}+\lambda \Delta t \mu_{j}\right. \\
& \left.\quad-\gamma\left(W_{N-1}-x_{N-1}\right)-\tilde{\eta}\left(W_{N-1}-x_{N-1}\right)\right)\left(W_{N-1}\right. \\
& \left.\left.\quad-x_{N-1}\right)\right] .
\end{aligned}
$$

Since the upper formula is a strictly concave function of $x_{N-1}$, we can obtain the optimal execution strategy by taking its partial derivative with respect to $x_{N-1}$ and solving for its zero

$$
x_{N-1}^{*}=\frac{W_{N-1}}{2}-\frac{\lambda \Delta t \mu_{j}}{2(\gamma+2 \widetilde{\eta})} .
$$

By substituting the optimal execution strategy into $V_{N-1}\left(S_{N-2}, W_{N-1}\right)$, the optimal-value function is as follows:

$$
\begin{aligned}
V_{N-1} & \left(S_{N-2}, W_{N-1}\right) \\
= & W_{N-1}\left(S_{N-2}-\frac{(3 \gamma+2 \widetilde{\eta})}{4} W_{N-1}+\frac{3 \lambda \triangle t \mu_{j}}{2}\right) \\
& +\frac{\left(\lambda \triangle t \mu_{j}\right)^{2}}{2(2 \gamma+4 \widetilde{\eta})} .
\end{aligned}
$$

Next, we set the optimal execution strategy and the optimalvalue function to be $x_{N-k}^{*}$ and $V_{N-k}$, respectively, where

$$
\begin{aligned}
& x_{N-k}^{*}=\frac{W_{N-k}}{k+1}-\frac{k \lambda \Delta t \mu_{j}}{2(\gamma+2 \widetilde{\eta})}, \quad k=0,1, \ldots, N-1, \\
& V_{N-k}\left(S_{N-k-1}, W_{N-k}\right)=W_{N-k}\left(S_{N-k-1}\right. \\
& \left.-\frac{(\gamma(k+2)+2 \widetilde{\eta})}{2(k+1)} W_{N-k}+\frac{(k+2) \lambda \Delta t \mu_{j}}{2}\right) \\
& +\frac{a_{k}\left(\lambda \Delta t \mu_{j}\right)^{2}}{2(2 \gamma+4 \widetilde{\eta})} .
\end{aligned}
$$

Let $k=k+1$; by using mathematical induction we may find

$$
\begin{aligned}
& V_{N-k-1}=\max _{x_{N-k-1}} \mathbb{E}_{N-k-1}\left[\widetilde{S}_{N-k-1} x_{N-k-1}\right. \\
& \left.+V_{N-k}\left(S_{N-k-1}, W_{N-k}\right)\right] \\
& \quad=\max _{x_{N-1}} \mathbb{E}_{N-k-1}\left[\left(S_{N-k-2}-\gamma x_{N-k-1}\right.\right. \\
& \left.+\sigma \sqrt{\triangle t} \varepsilon_{N-k-1}+\Delta J_{N-k-1}-\tilde{\eta} x_{N-k-1}\right) x_{N-k-1} \\
& +V_{N-k}\left(S_{N-k-2}-\gamma x_{N-k-1}+\sigma \sqrt{\Delta t} \varepsilon_{N-k-1}\right. \\
& \left.\left.+\triangle J_{N-k-1}, W_{N-k-1}-x_{N-k-1}\right)\right] .
\end{aligned}
$$

At time $N-k-1$, the conditional expectation of the above equation is

$$
\begin{aligned}
& V_{N-k-1}=\max _{x_{N-k-1}}\left[\left(S_{N-k-2}-\gamma x_{N-k-1}+\lambda \Delta t \mu_{j}\right.\right. \\
& \left.-\tilde{\eta} x_{N-k-1}\right) x_{T-k-1}+\left(S_{N-k-2}-\gamma x_{N-k-1}\right. \\
& +\lambda \Delta t \mu_{j}-\frac{(\gamma(k+2)+2 \widetilde{\eta})}{2(k+1)}\left(W_{N-k-1}-x_{N-k-1}\right) \\
& \left.+\frac{(k+2) \lambda \Delta t \mu_{j}}{2}\right)\left(W_{N-k-1}-x_{N-k-1}\right) \\
& \left.+\frac{a_{k}\left(\lambda \Delta t \mu_{j}\right)^{2}}{2(2 \gamma+4 \tilde{\eta})}\right] .
\end{aligned}
$$

Since the upper formula is a strictly concave function of $x_{N-k-1}$, we can obtain the optimal execution strategy by taking its partial derivative with respect to $x_{N-k-1}$ and solving for its zero

$$
x_{N-k-1}^{*}=\frac{W_{N-k-1}}{k+2}-\frac{(k+1) \lambda \Delta t \mu_{j}}{2(\gamma+2 \widetilde{\eta})} .
$$

By substituting the optimal execution strategy into $V_{N-k-1}$, the optimal-value function is as follows:

$$
\begin{aligned}
& V_{N-k-1}=W_{N-k-1}\left(S_{N-k-2}\right. \\
& -\frac{(\gamma((k+1)+2)+2 \widetilde{\eta})}{2((k+1)+1)} W_{N-k-1} \\
& \left.+\frac{((k+1)+2) \lambda \Delta t \mu_{j}}{2}\right) \\
& +\frac{\left(a_{(k+1)-1}+(1+(k+1))(k+1) / 2\right)\left(\lambda \Delta t \mu_{j}\right)^{2}}{2(2 \gamma+4 \widetilde{\eta})}
\end{aligned}
$$

This completes the induction. 


\section{B. Proof of (12)}

According to the definition of the distribution function, there are

$$
\begin{aligned}
& \Phi_{\Delta S_{t}}(x)=\mathbb{P}\left[\Delta S_{t} \leq x\right]=\mathbb{P}\left[\Delta G_{t}+\sum_{i=1}^{\Delta P_{t}} Y_{i} \leq x\right] \\
& =\sum_{k=0}^{\infty} \mathbb{P}\left[\Delta G_{t}+\sum_{i=1}^{\Delta P_{t}} Y_{i} \leq x \mid \Delta P_{t}=k\right] \mathbb{P}\left[\Delta P_{t}=k\right] \\
& =\sum_{k=0}^{\infty} p_{k}(\lambda \Delta t) \Phi^{(k)}(x),
\end{aligned}
$$

where $\Delta G_{t}=\sigma \Delta B_{t}$ is the incremental Gaussian process, $p_{k}(\lambda \triangle t)=(\lambda \triangle t)^{k} e^{-\lambda \Delta t} / k !, k=0,1, \ldots, \infty$ obeys the Poisson distribution with $\lambda \Delta t$. Set $\Phi^{(k)}(x) \equiv \mathbb{P}\left[\Delta G_{t}+\sum_{i=1}^{\Delta P_{t}} Y_{i} \leq x\right]$, $\triangle G_{t} \sim N\left(0, \sigma^{2} \triangle t\right)$, and based on convolution formula we can get

$$
\begin{aligned}
\Phi^{(k)}(x) & =\left(\Phi_{\triangle G_{t}} * \Psi_{Y}\right)(x) \\
& =\left(\Phi_{\triangle G_{t}} \prod_{i=1}^{k}\left(* \Psi_{Y_{i}}\right)\right)(x) \\
& =\left(\Phi_{\triangle G_{t}} *\left(\Psi_{Y}\right)^{k}\right)(x),
\end{aligned}
$$

where $\Psi_{Y_{i}} \sim N\left(\mu_{j}, \sigma_{j}^{2}\right)$ and $\Phi_{\triangle G_{t}}\left(* \Psi_{Y}\right)^{k}$ is the convolution of the incremental Gaussian process and $k$ number of random variables $\Psi_{Y_{i}}$ which is assumed to be independent and identically distributed. Hence, we can get

$$
\begin{aligned}
\Phi_{\triangle S_{t}}(x) & =\sum_{k=0}^{\infty} p_{k}(\lambda \triangle t)\left(\Phi_{\triangle G_{t}}\left(* \Psi_{Y}\right)^{k}\right)(x) \\
& =\sum_{k=0}^{\infty} p_{k}(\lambda \triangle t) \Phi\left(x ; k \mu_{j}, \sigma^{2} \Delta t+k \sigma_{j}^{2}\right),
\end{aligned}
$$

where $\Phi(x)$ is the accumulation normal distribution function. When $\triangle t \longrightarrow 0^{+}, \mathbb{P}\left[\Delta P_{t}=0\right]=1-\lambda \triangle t, \mathbb{P}\left[\Delta P_{t}=\right.$ 1] $=\lambda \triangle t$, and $\mathbb{P}\left[\Delta P_{t}>1\right]=\mathrm{o}(\Delta t)$. The probability density function of $\triangle S_{t}$ is

$$
\begin{aligned}
f_{\triangle S_{t}}(x)= & (1-\lambda \Delta t) N\left(x ; 0, \sigma^{2} \Delta t\right) \\
& +\lambda \Delta t N\left(x ; \mu_{j}, \sigma^{2} \Delta t+\sigma_{j}^{2}\right),
\end{aligned}
$$

with $\mathbb{E}\left(\Delta S_{t}\right)=\lambda \Delta t \mu_{j}$ and $\mathbb{D}\left(\triangle S_{t}\right)=\sigma^{2} \Delta t+\lambda \Delta t \sigma_{j}^{2}+$ $\lambda \triangle t(1-\lambda \triangle t) \mu_{j}^{2}$, where $N(\bullet)$ is the normal density function. The cumulative distribution function of $\triangle S_{t}$ is

$$
\Phi_{\triangle S_{t}}(x)=\int_{-\infty}^{x} f_{\triangle S_{t}}(y) d y
$$

\section{Proof of (13) and (14)}

In our model, a compound Poisson process is introduced into $\mathrm{ABM}$. Thus, the distribution of price increment depends on $\triangle t$, and we can obtain $\operatorname{VaR}_{\alpha}\left(\triangle S_{N}\right)=\operatorname{VaR}_{\alpha}\left(\triangle S_{N-1}\right)=\cdots=$ $\operatorname{VaR}_{\alpha}\left(\triangle S_{1}\right)=\Phi_{\triangle S_{1}}^{-1}(\alpha)$ with respect to (B.5). According to the iterative form of the value function of dynamic programming, we can get

$$
\begin{aligned}
& V_{k}\left(S_{k-1}, W_{k}\right)=\max _{x_{k}} \mathbb{E}_{k}\left[\widetilde{S}_{k} x_{k}-\operatorname{VaR}_{\alpha}\left(\triangle S_{1}\right) W_{k}\right. \\
& \left.\quad+V_{k+1}\left(S_{k}, W_{k+1}\right)\right] .
\end{aligned}
$$

By staring at the period $N$, we obtain

$$
\begin{aligned}
V_{N} & \left(S_{N-1}, W_{N}\right) \\
& =\max _{x_{N}} \mathbb{E}_{N}\left[\widetilde{S}_{N} W_{N}-V a R_{\alpha}\left(\triangle S_{1}\right) W_{N}\right] \\
& =\left(S_{N-1}-\gamma W_{N}+\lambda \Delta t \mu_{j}-\widetilde{\eta} W_{N}-V a R_{\alpha}\left(\triangle S_{1}\right)\right) \\
& \cdot W_{N} .
\end{aligned}
$$

Since this function must satisfies $W_{N+1}=0$, there is no choice but to execute the entire remaining order $W_{N}$ at period $N$. In the next-to-last period $N-1$, the Bellman iteration is as follows:

$$
\begin{aligned}
& V_{N-1}\left(S_{N-2}, W_{N-1}\right)=\max _{x_{N-1}} \mathbb{E}_{N-1}\left[\widetilde{S}_{N-1} x_{N-1}\right. \\
& \left.\quad-\operatorname{VaR}_{\alpha}\left(\triangle S_{1}\right) W_{N-1}+V_{N}\left(S_{N-1}, W_{N}\right)\right] \\
& \quad=\max _{x_{N-1}} \mathbb{E}_{N-1} \max \left[\left(S_{N-2}-\gamma x_{N-1}+\sigma \sqrt{\Delta t} \varepsilon_{N-1}\right.\right. \\
& \left.+\triangle J_{N-1}-\widetilde{\eta} x_{N-1}\right) x_{N-1} \\
& -V a R_{\alpha}\left(\triangle S_{1}\right) W_{N-1}+V_{N}\left(S_{N-2}-\gamma x_{N-1}\right. \\
& \left.\left.+\sigma \sqrt{\triangle t} \varepsilon_{N-1}+\Delta J_{N-1}, W_{N-1}-x_{N-1}\right)\right] .
\end{aligned}
$$

The conditional expectation of the above equation at period $N-1$ is

$$
\begin{aligned}
& V_{N-1}\left(S_{N-2}, W_{N-1}\right)=\max _{x_{N-1}}\left[\left(S_{N-2}-\gamma x_{N-1}+\lambda \Delta t \mu_{j}\right.\right. \\
& \left.-\tilde{\eta} x_{N-1}\right) x_{N-1}-\operatorname{VaR}_{\alpha}\left(\triangle S_{1}\right) W_{N-1}+\left(S_{N-2}\right. \\
& -\gamma x_{N-1}+\lambda \Delta t \mu_{j}-\gamma\left(W_{N-1}-x_{N-1}\right)+\lambda \Delta t \mu_{j} \\
& \left.-\tilde{\eta}\left(W_{N-1}-x_{N-1}\right)-V_{a}\left(\triangle S_{1}\right)\right)\left(W_{N-1}\right. \\
& \left.\left.-x_{N-1}\right)\right] .
\end{aligned}
$$


Since the upper formula is a strictly concave function of $x_{N-1}$, we can obtain the optimal execution strategy by taking its partial derivative with respect to $x_{N-1}$ and solving for its zero:

$$
x_{N-1}^{*}=\frac{W_{N-1}}{2}-\frac{\lambda \Delta t \mu_{j}-V a R_{\alpha}\left(\triangle S_{1}\right)}{2(\gamma+2 \widetilde{\eta})} .
$$

By substituting the optimal execution strategy into $V_{N-1}\left(S_{N-2}, W_{N-1}\right)$, the optimal-value function is as follows:

$$
\begin{aligned}
& V_{N-1}\left(S_{N-2}, W_{N-1}\right)=W_{N-1}\left(S_{N-2}\right. \\
& \left.-\frac{(3 \gamma+2 \widetilde{\eta})}{4} W_{N-1}+\frac{3\left(\lambda \triangle t \mu_{j}-V a R_{\alpha}\left(\triangle S_{1}\right)\right)}{2}\right) \\
& +\frac{\left(\lambda \triangle t \mu_{j}-V a R_{\alpha}\left(\triangle S_{1}\right)\right)^{2}}{2(2 \gamma+4 \widetilde{\eta})} .
\end{aligned}
$$

Next, we set the optimal execution strategy and the optimalvalue function to be $x_{N-k}^{*}$ and $V_{N-k}$, respectively, where

$$
\begin{aligned}
& x_{N-k}^{*}= \frac{W_{N-k}}{k+1}-\frac{k\left(\lambda \triangle \mu_{j}-V a R_{\alpha}\left(\Delta S_{1}\right)\right)}{2(\gamma+2 \widetilde{\eta})}, \\
& k=0,1, \ldots, N-1, \\
& V_{N-k}=W_{N-k}\left(S_{N-k-1}-\frac{(\gamma(k+2)+2 \widetilde{\eta})}{2(k+1)} W_{N-k}\right. \\
&\left.+\frac{(k+2)\left(\lambda \triangle t \mu_{j}-V a R_{\alpha}\left(\triangle S_{1}\right)\right)}{2}\right) \\
&+\frac{a_{k}\left(\lambda \triangle t \mu_{j}-V a R_{\alpha}\left(\triangle S_{1}\right)\right)^{2}}{2(2 \gamma+4 \widetilde{\eta})} .
\end{aligned}
$$

Let $k=k+1$, and $k>0$; by using mathematical induction we may find

$$
\begin{aligned}
& V_{N-k-1}=\max _{x_{N-k-1}} \mathbb{E}_{N-k-1}\left[\widetilde{S}_{N-k-1} x_{N-k-1}-\operatorname{VaR}_{\alpha}\left(\Delta S_{1}\right)\right. \\
& \left.\cdot W_{N-k-1}+V_{N-k}\left(S_{N-k-1}, W_{N-k}\right)\right] \\
& =\max _{x_{N-k-1}} \mathbb{E}_{N-k-1}\left[\left(S_{N-k-2}-\gamma x_{N-k-1}\right.\right. \\
& \left.+\sigma \sqrt{\Delta t} \varepsilon_{N-k-1}+\Delta J_{N-k-1}-\tilde{\eta} x_{N-k-1}\right) x_{N-k-1} \\
& -V a R_{\alpha}\left(\triangle S_{1}\right) W_{N-k-1}+V_{N-k}\left(S_{N-k-2}-\gamma x_{N-k-1}\right. \\
& \left.\left.+\sigma \sqrt{\triangle t} \varepsilon_{N-k-1}+\Delta J_{N-k-1}, W_{N-k-1}-x_{N-k-1}\right)\right] .
\end{aligned}
$$

The conditional expectation of the above equation at $N-k-1$ is

$$
\begin{aligned}
& V_{N-k-1}=\max _{x_{N-k-1}}\left[\left(S_{N-k-2}-\gamma x_{N-k-1}+\lambda \Delta t \mu_{j}\right.\right. \\
& \left.-\tilde{\eta} x_{N-k-1}\right) x_{N-k-1}-V a R_{\alpha}\left(\Delta S_{N-k-1}\right) W_{N-k-1} \\
& +\left(S_{N-k-2}-\gamma x_{N-k-1}+\lambda \Delta t \mu_{j}\right. \\
& -\frac{(\gamma(k+2)+2 \widetilde{\eta})}{2(k+1)}\left(W_{N-k-1}-x_{N-k-1}\right) \\
& \left.+\frac{(k+2)\left(\lambda \Delta t \mu_{j}-V a R_{\alpha}\left(\Delta S_{1}\right)\right)}{2}\right)\left(W_{N-k-1}\right. \\
& \left.\left.-x_{N-k-1}\right)+\frac{a_{k}\left(\lambda \Delta t \mu_{j}-V a R_{\alpha}\left(\Delta S_{1}\right)\right)^{2}}{2(2 \gamma+4 \widetilde{\eta})}\right] .
\end{aligned}
$$

Since the upper formula is a strictly concave function of $x_{N-k-1}$, we can obtain the optimal execution strategy by taking its partial derivative with respect to $x_{N-k-1}$ and solving for its zero:

$$
\begin{aligned}
x_{N-k-1}^{*}= & \frac{W_{N-k-1}}{k+2} \\
& -\frac{(k+1)\left(\lambda \triangle t \mu_{j}-V a R_{\alpha}\left(\triangle S_{1}\right)\right)}{2(\gamma+2 \widetilde{\eta})} .
\end{aligned}
$$

By substituting the optimal execution strategy into $V_{N-k-1}$, the optimal-value function is as follows:

$$
\begin{aligned}
V_{N-k-1}= & W_{N-k-1}\left(S_{N-k-2}-\frac{(\gamma((k+1)+2)+2 \widetilde{\eta})}{2((k+1)+1)} W_{N-k-1}+\frac{((k+1)+2)\left(\lambda \Delta t \mu_{j}-V a R_{\alpha}\left(\Delta S_{1}\right)\right)}{2}\right) \\
& +\frac{\left(a_{(k+1)-1}+(1+(k+1))(k+1) / 2\right)\left(\lambda \Delta t \mu_{j}-V a R_{\alpha}\left(\Delta S_{1}\right)\right)^{2}}{2(2 \gamma+4 \widetilde{\eta})} .
\end{aligned}
$$

This completes the induction. 


\section{Data Availability}

The data used to support the findings of this study are available from the corresponding author upon request.

\section{Conflicts of Interest}

The authors declare that there are no conflicts of interest regarding the publication of this paper.

\section{References}

[1] A. F. Perold, "The implementation shortfall," The Journal of Portfolio Management, vol. 14, no. 3, pp. 4-9, 1988.

[2] F. J. Fabozzi, The handbook of financial instruments, John Wiley Sons, 2003.

[3] S. Moazeni, T. F. Coleman, and Y. Li, "Optimal execution under jump models for uncertain price impact," The Journal of Computational Finance, vol. 16, no. 4, pp. 35-78, 2013.

[4] D. Bertsimas and A. W. Lo, "Optimal control of execution costs," Journal of Financial Markets, vol. 1, no. 1, pp. 1-50, 1998.

[5] R. Almgren and N. Chriss, "Value under liquidation," Risk, vol. 12, no. 12, pp. 61-63, 1999.

[6] R. Almgren and N. Chriss, "Optimal execution of portfolio transactions," The Journal of Risk, vol. 3, no. 2, pp. 5-39, 2001.

[7] A. Schied and T. Schöneborn, "Risk aversion and the dynamics of optimal liquidation strategies in illiquid markets," Finance and Stochastics, vol. 13, no. 2, pp. 181-204, 2009.

[8] J. Gatheral and A. Schied, "Optimal trade execution under geometric Brownian motion in the Almgren and Chriss framework," International Journal of Theoretical and Applied Finance, vol. 14, no. 3, pp. 353-368, 2011.

[9] A. Schied, "Robust strategies for optimal order execution in the Almgren-Chriss framework," Applied Mathematical Finance, vol. 20, no. 3, pp. 264-286, 2013.

[10] P. A. Forsyth, "A Hamilton-Jacobi-Bellman approach to optimal trade execution," Applied Numerical Mathematics, vol. 61, no. 2 , pp. 241-265, 2011.

[11] P. A. Forsyth, J. S. Kennedy, S. T. Tse, and H. Windcliff, "Optimal trade execution: a mean quadratic variation approach," Journal of Economic Dynamics \& Control, vol. 36, no. 12, pp. 1971-1991, 2012.

[12] X. Cheng, M. Di Giacinto, and T.-H. Wang, "Optimal execution with uncertain order fills in Almgren-Chriss framework," Quantitative Finance, vol. 17, no. 1, pp. 55-69, 2017.

[13] G. Huberman and W. Stanzl, "Optimal liquidity trading," Review of Finance, vol. 9, no. 2, pp. 165-200, 2005.

[14] C. M. Ahn and H. E. Thompson, "Jump-diffusion processes and the term structure of interest rates," The Journal of Finance, vol. 43, no. 1, pp. 155-174, 1988.

[15] Y. Aït-Sahalia, "Telling from discrete data whether the underlying continuous-time model is a diffusion," Journal of Finance, vol. 57, no. 5, pp. 2075-2112, 2002.

[16] Y. Aït-Sahalia, "Disentangling diffusion from jumps," Journal of Financial Economics, vol. 74, no. 3, pp. 487-528, 2004.

[17] R. Cont, "Empirical properties of asset returns: stylized facts and statistical issues," Quantitative Finance, vol. 1, pp. 223-236, 2001.

[18] Y. Aït-Sahalia, J. Cacho-Diaz, and R. J. A. Laeven, "Modeling financial contagion using mutually exciting jump processes," Journal of Financial Economics, vol. 117, no. 3, pp. 585-606, 2015.
[19] F. M. Bandi and R. Renò, "Price and volatility co-jumps," Journal of Financial Economics, vol. 119, no. 1, pp. 107-146, 2016.

[20] R. Bellman, Dynamic Programming, Princeton University Press, Princeton, NJ, USA, 1957. 


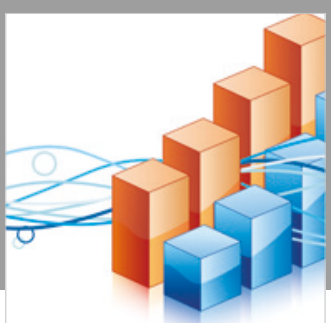

Advances in

Operations Research

\section{-n-m}
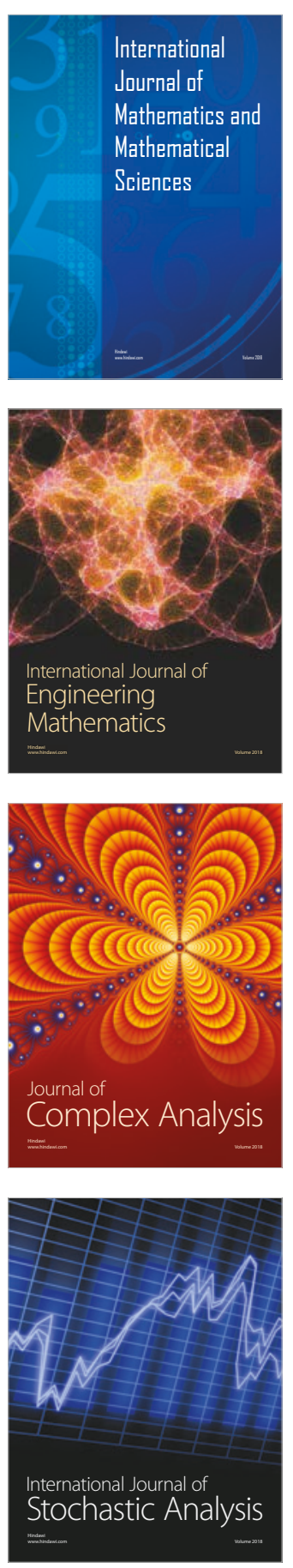
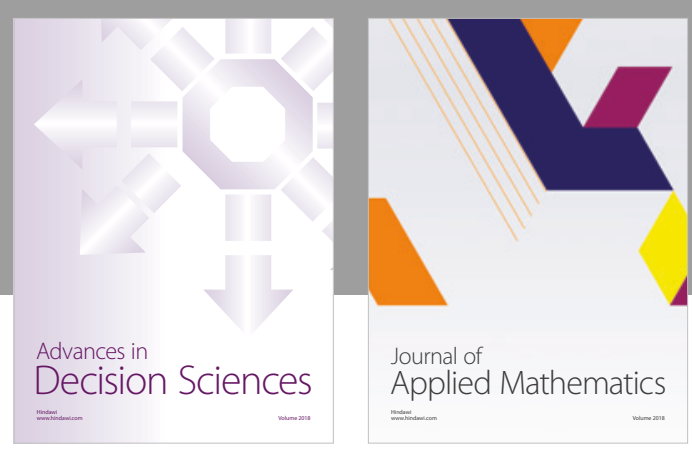

Journal of

Applied Mathematics
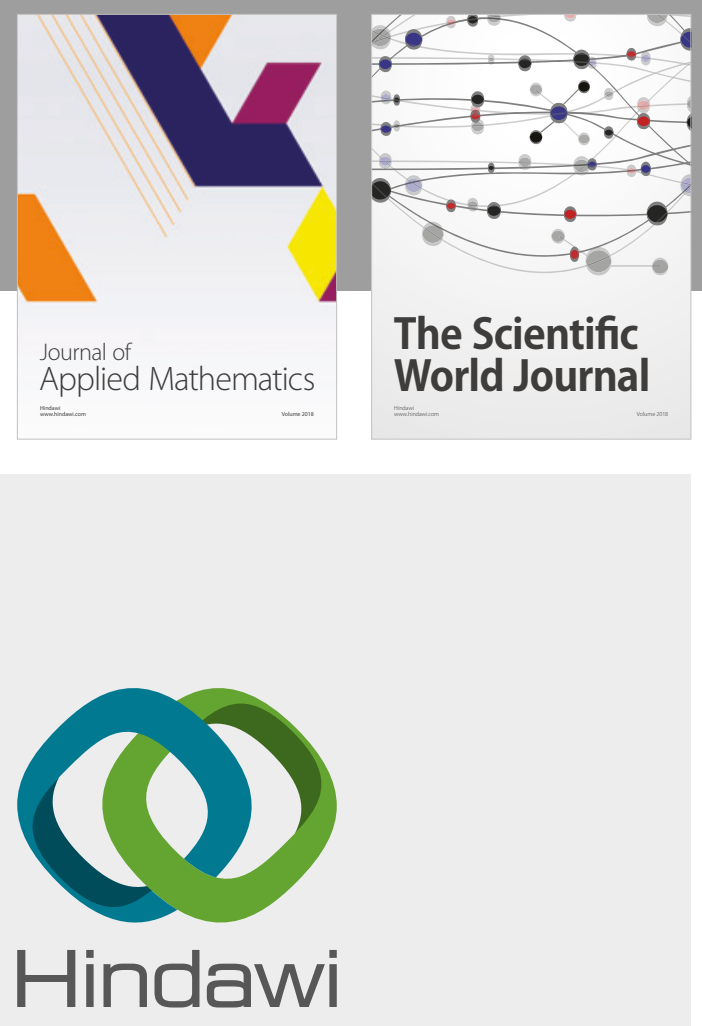

Submit your manuscripts at

www.hindawi.com

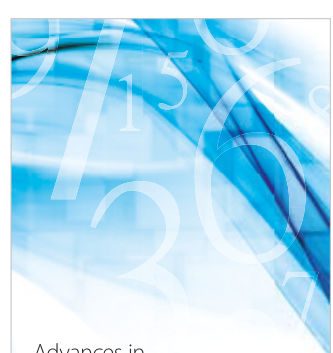

Advances in
Numerical Analysis
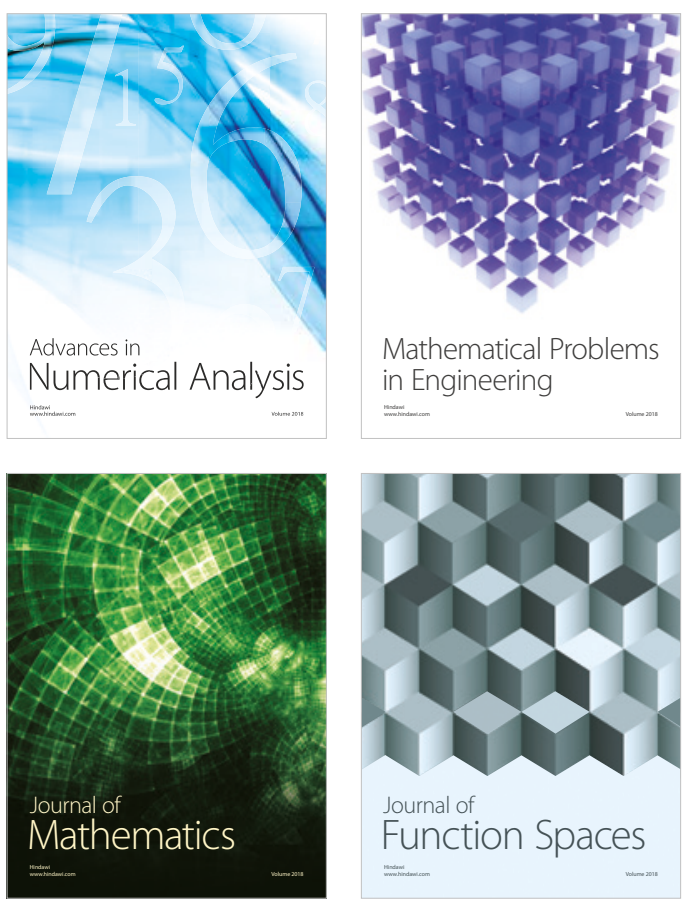

Mathematical Problems in Engineering

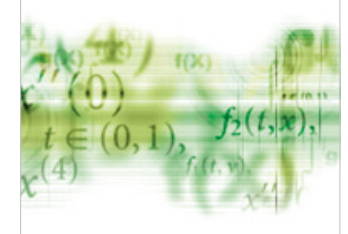

International Journal of

Differential Equations

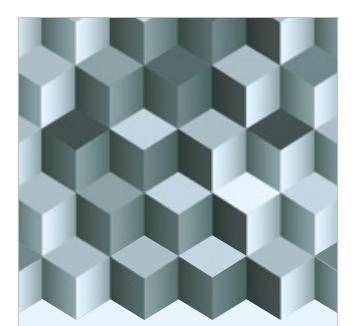

Journal of

Function Spaces

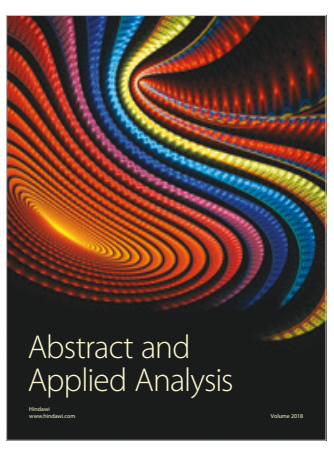

The Scientific

World Journal

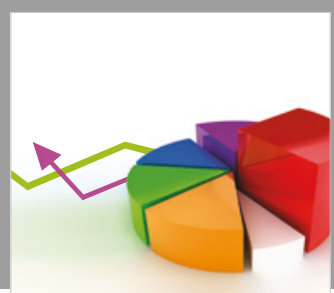

Journal of

Probability and Statistics
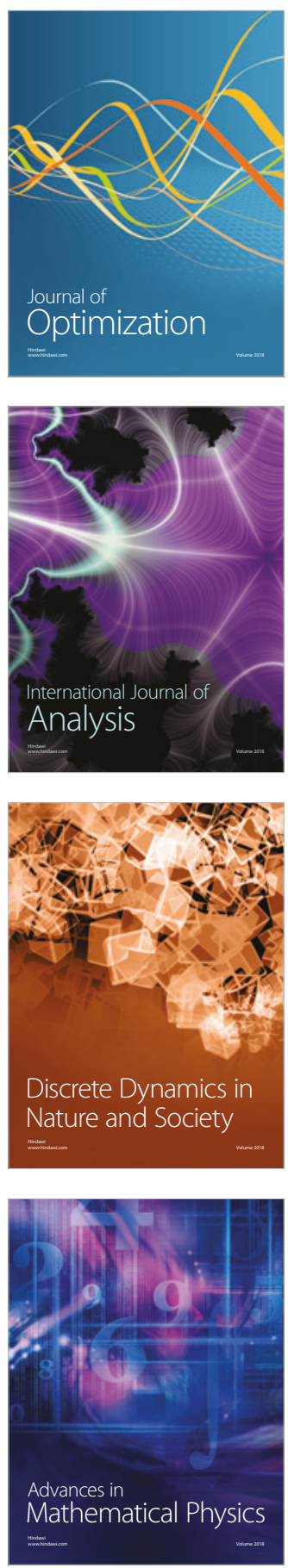